A Mamum and F Willekens for their collaboration in the understanding of the life table approach.

Contributors: All authors participated actively in conception and design of the study or analysis and interpretation of data, in drafting the article or revising it critically for important intellectual content, and in final approval of the version to be published. $\mathrm{OHF}$ is the guarantor.

Funding: This study was supported by grants from the Netherlands Heart Foundation (grant no 98.138) and the Netherlands Organization for Scientific Research (grant no 904-66-093). OHF, LB, CdL, AP, EWS, and JPM were partly funded by the Netherlands Heart Foundation (grant no 98.138) and the Netherlands Organization for Scientific Research (grant no 904-66-093). AP was also partly funded by VicHealth (fellowship grant no 2002-0191). All authors have acted independently from the funders of this project.

Competing interests: None declared.

Ethical approval: Not needed as this was a secondary data analysis.

1 Gluckman TJ, Baranowski B, Ashen MD, Henrikson CA, McAllister M, Braunstein JB, et al. A practical and evidence-based approach to cardioBraunstein JB, et al. A practical and evidence-based approach to car

2 Wald NJ, Law MR. A strategy to reduce cardiovascular disease by more than $80 \%$. BMJ 2003;326:1419-23.

3 Correspondence. "Polypill" to fight cardiovascular disease. BMJ 2003;327:807-10.

4 Sacket DL, Straus SE, Richardson WS, Rosenberg W, Haynes RB Evidence-based medicine: how to practice and teach EBM. New York: Churchill Livingstone, 2000.

5 Oxford Centre for Evidence Based Medicine. Levels of evidence and grades of recommendation. www.cebm.net/levels_of_evidence.asp (accessed 5 Aug 2004)

6 Di Castelnuovo A, Rotondo S, Iacoviello L, Donati MB, De Gaetano G. Meta-analysis of wine and beer consumption in relation to vascular risk. Circulation 2002;105:2836-44

7 Whelton SP, He J, Whelton PK, Muntner P. Meta-analysis of observational studies on fish intake and coronary heart disease. Am I Cardiol 2004;93:1119-23.
Taubert D, Berkels R, Roesen R, Klaus W. Chocolate and blood pressure in elderly individuals with isolated systolic hypertension. JAMA 2003;290:1029-30.

9 Neal B, MacMahon S, Chapman N. Effects of ACE inhibitors, calcium antagonists, and other blood-pressure-lowering drugs: results of prospectively designed overviews of randomised trials. Lancet 2000;356:1955-64

10 John JH, Ziebland S, Yudkin P, Roe LS, Neil HA. Effects of fruit and vegetable consumption on plasma antioxidant concentrations and blood pressure: a randomised controlled trial. Lancet 2002;359:1969-74.

11 Ackermann RT, Mulrow CD, Ramirez G, Gardner CD, Morbidoni L, Lawrence VA. Garlic shows promise for improving some cardiovascular risk factors. Arch Intern Med 2001;161:813-24.

12 Silagy C, Neil A. Garlic as a lipid lowering agent-a meta-analysis. J $R$ Coll Physicians Lond 1994;28:39-45.

13 Law MR, Wald NJ, Thompson SG. By how much and how quickly does reduction in serum cholesterol concentration lower risk of ischaemic heart disease? BMJ 1994;308:367-72

14 Berthold HK, Sudhop T, von Bergmann K. Effect of a garlic oil preparation on serum lipoproteins and cholesterol metabolism: a randomized controlled trial. JAMA 1998;279:1900-2.

15 Jenkins DJ, Kendall CW, Marchie A, Parker TL, Connelly PW, Qian W, et al. Dose response of almonds on coronary heart disease risk factors: blood lipids, oxidized low-density lipoproteins, lipoprotein(a) homocysteine, and pulmonary nitric oxide: a randomized, controlled, crossover cysteine, and pulmonary nitric oxide:

16 Sabate J, Haddad E, Tanzman JS, Jambazian P, Rajaram S. Serum lipid response to the graduated enrichment of a step I diet with almonds: a
randomized feeding trial. Am J Clin Nutr 2003;77:1379-84.

17 Dawber TR, Meadors GF, Moore FE Jr. Epidemiological approaches to heart disease: the Framingham study. Am J Public Health 1951;41:279-81.

18 Stokes J 3rd, Kannel WB, Wolf PA, Cupples LA, D'Agostino RB. The relative importance of selected risk factors for various manifestations of cardiovascular disease among men and women from 35 to 64 years old: 30 years of follow-up in the Framingham study. Circulation 1987;75:V65-73.

19 De Backer G, Ambrosioni E, Borch-Johnsen K, Brotons C, Cifkova R, Dallongeville J, et al. European guidelines on cardiovascular disease prevention in clinical practice. Eur Heart J 2003:24:1601-10.

20 Peers A, MamunAA, Willekens, Bonne L A cadiovalo life history: a life course analysis of the original Framingham heart study cohort. Eur Heart J 2002;23:458-66.

21 US Department of Health and Human Services. Physical activity and health: a report of the surgeon general. Atlanta: Centers for Disease Control and Prevention, National Center for Chronic Disease Prevention and Health Promotion, 1996

(Accepted 4 November 2004)

\title{
Randomised controlled trial of magnetic bracelets for relieving pain in osteoarthritis of the hip and knee
}

College Surgery, Cullompton, Devon

EX15 1TG

Tim Harlow

general practitioner

Peninsula Medical

School (Primary

Care), Exeter

EX2 5DW

Colin Greaves

research fellow

Peninsula Medical

School, Tamar

Science Park,

Plymouth PL6 8BX

Adrian White

senior research fellow

Peninsula Medical

School

(Complementary

Medicine), Exeter

EX2 4NT

Liz Brown

research assistant

Edzard Ernst

professor of

complementary

medicine

continued over

BMJ 2004;329:1450-4

Tim Harlow, Colin Greaves, Adrian White, Liz Brown, Anna Hart, Edzard Ernst

\section{Abstract}

Objective To determine the effectiveness of commercially available magnetic bracelets for pain control in osteoarthritis of the hip and knee.

Design Randomised, placebo controlled trial with three parallel groups.

Setting Five rural general practices.

Participants 194 men and women aged 45-80 years with osteoarthritis of the hip or knee.

Intervention Wearing a standard strength static bipolar magnetic bracelet, a weak magnetic bracelet, or a non-magnetic (dummy) bracelet for 12 weeks. Main outcome measures Change in the Western Ontario and McMaster Universities osteoarthritis lower limb pain scale (WOMAC A) after 12 weeks, with the primary comparison between the standard and dummy groups. Secondary outcomes included changes in WOMAC B and C scales and a visual analogue scale for pain.

Results Mean pain scores were reduced more in the standard magnet group than in the dummy group (mean difference 1.3 points, $95 \%$ confidence interval
0.05 to 2.55 ). Self reported blinding status did not affect the results. The scores for secondary outcome measures were consistent with the WOMAC A scores. Conclusion Pain from osteoarthritis of the hip and knee decreases when wearing magnetic bracelets. It is uncertain whether this response is due to specific or non-specific (placebo) effects.

Manufacturers of permanent static magnet devices claim that they reduce pain in various conditions, including osteoarthritis. ${ }^{1}$ Worldwide sales were estimated at \$5bn (£2.6bn, €3.8bn) in 1999. ${ }^{2}$ Osteoarthritis affects around 760000 people in the United Kingdom, producing an estimated 3.02 million general practice consultations in 2000. If magnets were effective they would offer a cheap and probably safe treatment option.

Some studies of permanent static magnets have found significant pain reduction ${ }^{2-9}$ whereas others reported no effect. ${ }^{10-12}$ Major differences exist in the type and strength of magnets used, the conditions

A chart showing flow of participants is on bmj.com 


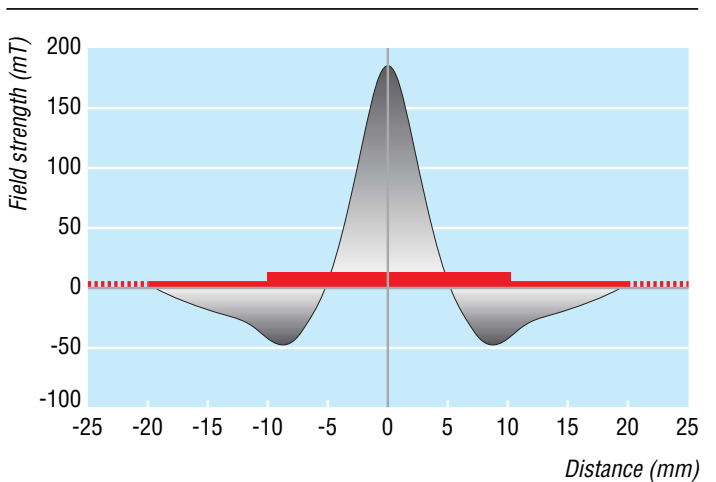

Magnetic field strength (mTesla) across the surface of a standard magnetic bracelet (20 $\mathrm{mm}$ diameter)

treated, and treatment times. There are also methodological concerns about small sample size and difficulties in maintaining blinding. ${ }^{2}$ We therefore aimed to conduct an adequately powered trial testing the hypothesis that magnetic bracelets, as used in the consumer market, reduce pain in osteoarthritis of the hip and knee.

\section{Participants and methods}

Between December 2001 and December 2003, we recruited 194 participants aged 45-80 years with osteoarthritis of the hip or knee from five rural general practices in Mid Devon (see bmj.com). Osteoarthritis was diagnosed by a consultant (orthopaedic surgeon or rheumatologist) or a general practitioner, and we sought confirmatory radiological evidence for participants who had none recorded in their general practice notes. Participants had to score 8-20 points on the Western Ontario and McMaster Universities osteoarthritis index (WOMAC A) on entry. ${ }^{13}{ }^{14}$ We excluded people with a cardiac pacemaker, current magnetic bracelet, surgery to the index joint (excluding arthroscopy), or haemophilia and women who were pregnant or breast feeding.

Recruitment was by referral from doctors, advertising, or invitation after a search of practice records. Trial nurses arranged radiological confirmation of diagnosis if needed, and they collected data in surgery based clinics at 0,4 , and 12 weeks. Participants were given a full strength bracelet at the end of the trial.

\section{Intervention and randomisation}

The participants, trial nurse, and healthcare providers, were blinded to treatment allocation. Treatments consisted of identical looking bracelets containing three different components. The manufacturer's specifications were:

Group A-Standard neodymium magnets set in a steel backing cup, with the open side facing the ventral wrist, creating a fluctuating magnetic pattern across the bracelet (fig 1). The field strength at the wrist contact surface was $170-200$ mTesla.

Group B-Weak magnets with no backing plate. The field was strong enough to seem magnetic on testing (21-30 mTesla), but previous research suggests this is insufficient to be therapeutic. ${ }^{15}$ This was intended to provide an undetectable placebo.

Group C-Non-magnetic steel washers.
The National Physical Laboratory tested five bracelets of each type before the study, confirming the manufacturer's specification.

An independent researcher randomised participants in blocks of 15 (five of each bracelet type per block), using random numbers generated in Microsoft Excel. A decode sheet was sealed and locked away. A second researcher checked the procedure. On enrolment, participants were told that they would receive either an active or an inactive bracelet.

\section{Outcome measures}

The predefined primary outcome measure was change in WOMAC A score after 12 weeks' follow up..$^{13}$ Secondary outcomes were a visual analogue scale asking, "How bad was the pain from your arthritis in the last week when it was at its worst?" with verbal and numerical anchors from none (0) to worst imaginable (100) ${ }^{16}$; WOMAC B and C scores, measuring leg stiffness and functioning ${ }^{13}{ }^{14}$; the number of days participants had used analgesics in the past week; and perceived monetary value of the bracelet.

We assessed compliance with wearing the bracelet at 4 and 12 weeks using a visual analogue scale. Blinding was assessed at 12 weeks by asking whether participants thought they had an active bracelet and the reason for such belief.

The estimated effect size was based on a $20 \%$ differential reduction in WOMAC A score, which was considered commensurate with effect sizes in studies of analgesics and osteoarthritis. ${ }^{13}{ }^{17}$ A sample size of 52 in each of the groups would have $80 \%$ power to detect a difference in one way analysis of variance of change scores, assuming mean changes of 3, 1.5, and 1 and a common standard deviation of 3.4. Assuming 15\% dropout, we planned to recruit 64 subjects to each group. ${ }^{18}$ We checked the suitability of these numbers for an analysis of variance across the three groups by using a range of estimated small average changes for the weak magnet group.

\section{Analysis}

The analysis was specified in advance of the study as follows. Last value carried forward was used to impute missing values for subsequent visits. The blinded statistician conducted analysis of variance on all three groups using SPSS version 11.5, with change in WOMAC A score at 12 weeks as the response. The robustness of the results was checked with analysis of covariance on the WOMAC A score at 12 weeks with baseline WOMAC A as covariate, and checking sensitivity to baseline imbalances. Dunnett's test was then used to compare the means for the dummy and weak magnet group separately with the mean for the standard magnet group. The protocol specified that

Table 1 Baseline characteristics of participants randomised to standard, weak, and dummy magnetic bracelets.

\begin{tabular}{lccc} 
Group & $\begin{array}{c}\text { Standard } \\
(\mathbf{n}=65)\end{array}$ & $\begin{array}{c}\text { Weak } \\
(\mathbf{n}=64)\end{array}$ & $\begin{array}{c}\text { Dummy } \\
(\mathbf{n}=64)\end{array}$ \\
\hline Mean (SD) age (years) & $66.6(8.4)$ & $66.8(8.3)$ & $66.3(9.1)$ \\
\hline Mean (SD) height (m) & $1.69(0.11)$ & $1.67(0.11)$ & $1.67(0.09)$ \\
\hline Mean (SD) weight (kg) & $86.7(20.3)$ & $82.4(17.1)^{*}$ & $82.7(16.4)$ \\
\hline No (\%) of men & $34(52)$ & $46(72)$ & $39(61)$ \\
\hline $\begin{array}{l}\text { Median (interquartile range) No of days } \\
\text { in past week when painkillers used }\end{array}$ & $5.5(1$ to 7$)$ & $6.5(2$ to 7$)$ & $7.0(1 \text { to } 7)^{*}$ \\
\hline${ }^{*} \mathrm{n}=63$. & & &
\end{tabular}


the primary comparison was dummy versus standard magnets, the other comparison being secondary, unless a high degree of unblinding was observed. Models were checked by examination of residuals and sensitivity to imputed values.

Subsequent analyses were unblinded. We used general linear models on all subjects to explore the association between outcomes and magnetic strength of individual bracelets. Similar analyses were then carried out, where appropriate, for WOMAC B and C and the global pain score.

\section{Results}

\section{Response rates and sample properties}

Of the 391 people assessed for eligibility, $144 \mathrm{did}$ not satisfy the inclusion criteria and $194(78.5 \%)$ of the remaining 247 accepted entry into the trial (see bmj.com). Group baseline characteristics were similar (table 1). Very few participants were lost to follow up. These were evenly spread across the three groups, and their baseline WOMAC A scores were not markedly different from those of participants with complete data. Reported compliance was high, with most wearing the bracelets for $100 \%$ of waking hours.

After the trial, we tested all the returned bracelets using a calibrated Hall effect probe. This showed that the standard magnets had a mean strength of 186 (range 134-197) mTesla (only one was outside the specified range) and the non-magnetic group all had zero strength. Because of a manufacturing error, only 28 of the weak magnets were within the specified range (21-30 mTesla). The mean for these 28 magnets was 26 mTesla; 34 magnets had a strength of 69-196 (mean 128) mTesla, and two were not returned (these were assumed to be in the specified range as they were part of a good batch).

\section{Analysis of outcomes}

Table 2 shows the scores for the three groups at baseline and after 4 and 12 weeks. Analysis of variance between the three groups on the change in WOMAC A from baseline to 12 weeks showed a difference that was just non-significant $(\mathrm{F}=2.90, \mathrm{df}=2,190 ; \mathrm{P}=0.057)$. Results from analysis of covariance on the score at 12 weeks (with baseline WOMAC A score entered as a covariate $)$ were significant $(\mathrm{F}=3.24, \mathrm{df}=2,189$; $\mathrm{P}=0.041)$.

The planned comparison (Dunnett's test) showed a significant mean difference in change in WOMAC A score of 1.3 between the standard and dummy magnet groups $(95 \%$ confidence interval 0.09 to $2.60 ; \mathrm{P}=0.03)$, but not between the standard and weak groups (mean difference $0.81,-0.44$ to $2.07 ; \mathrm{P}=0.26$ ). A similar pattern was observed for the change in WOMAC $\mathrm{C}$ score. The overall analysis of variance gave significant results $(\mathrm{F}=4.45, \mathrm{df}=2,190 ; \mathrm{P}=0.013)$, and Dunnett's test showed a significant mean difference between the standard and dummy groups $(4.4,95 \%$ confidence interval 1.0 to $7.9 ; \mathrm{P}=0.01$ ) but not between the standard and weak groups $(3.3,-0.2$ to $6.7 ; \mathrm{P}=0.07)$. Analysis of the visual analogue pain score showed a significant mean difference between the standard and dummy groups of 11.4 (95\% confidence interval 3.0 to 19.8). Change in WOMAC B scores did not differ
Table 2 Mean (SD) scores at baseline and 4 and 12 weeks by randomisation to standard, weak, and dummy magnetic bracelet

\begin{tabular}{|c|c|c|c|}
\hline $\begin{array}{l}\text { Outcome } \\
\text { measure }\end{array}$ & $\begin{array}{c}\text { Standard } \\
(n=65)\end{array}$ & $\begin{array}{c}\text { Weak } \\
(n=64)\end{array}$ & $\begin{array}{c}\text { Dummy } \\
(n=64)\end{array}$ \\
\hline \multicolumn{4}{|l|}{ WOMAC A: } \\
\hline Baseline & $10.7(2.1)$ & $11.0(2.0)$ & $10.9(2.1)$ \\
\hline 4 weeks & $8.9(3.8) \mathrm{m}=2$ & $9.1(2.8) \mathrm{m}=2$ & 9.5 (3.1) $m=0$ \\
\hline 12 weeks & 7.8 (3.9) $m=3$ & $8.8(3.2) m=5$ & 9.3 (3.2) $\mathrm{m}=3$ \\
\hline \multicolumn{4}{|l|}{ WOMAC B: } \\
\hline Baseline & $4.5(1.3)$ & $4.5(1.5)$ & $4.6(1.1)$ \\
\hline 4 weeks & $3.8(1.5) \mathrm{m}=2$ & $4.2(1.4) \mathrm{m}=2$ & $4.2(1.1) \mathrm{m}=0$ \\
\hline 12 weeks & $3.7(1.7) \mathrm{m}=3$ & $3.9(1.6) \mathrm{m}=5$ & $4.1(1.3) \mathrm{m}=3$ \\
\hline \multicolumn{4}{|l|}{ WOMAC C: } \\
\hline Baseline & $36.0(9.8)$ & $35.5(10.2)$ & $35.2(9.5)$ \\
\hline 4 weeks & $32.5(12.2) \mathrm{m}=2$ & 32.5 (11.6) $\mathrm{m}=2$ & $33.5(10.5) \mathrm{m}=0$ \\
\hline 12 weeks & 29.0 (13.1) $\mathrm{m}=3$ & $31.8(12.5) \mathrm{m}=5$ & $32.7(11.1) \mathrm{m}=3$ \\
\hline \multicolumn{4}{|c|}{ Pain on visual analogue scale: } \\
\hline Baseline & $66.8(16.2)$ & $64.9(18.3)$ & $63.5(18.3)$ \\
\hline 4 weeks & $61.8(20.0) \mathrm{m}=2$ & 60.2 (19.4) $m=2$ & $60.2(17.6) \mathrm{m}=0$ \\
\hline 12 weeks & $54.8(24.5) \mathrm{m}=3$ & $55.7(22.2) \mathrm{m}=5$ & $62.9(22.2) \mathrm{m}=3$ \\
\hline
\end{tabular}

WOMAC=Western Ontario and MacMaster University osteoarthritis index. $\mathrm{m}=$ number of values imputed from last recorded figure.

between groups $(\mathrm{F}=0.73, \mathrm{df}=2,190 ; \mathrm{P}=0.48)$. No important differences in these results emerged when either sex or analgesic use (at 12 weeks) was included as a covariate.

Table 3 provides data on participants' beliefs about group allocation and the reasons given for their beliefs. Around a third of participants in the standard and dummy groups were correct in their beliefs about their bracelet, although the reasons differed between groups. In the standard group beliefs were mainly based on noticing the magnetic force-for example, bracelets were often reported to stick to keys in pockets-or on improved symptoms. In the dummy group, few noticed the magnetic force and beliefs were most commonly based on a lack of symptom improvement.

Comparing the outcomes for the different belief groups is not appropriate because belief may follow benefit or lack of it, and any differences would therefore be hard to interpret. ${ }^{19}$ However, we have a more direct way of estimating the effect of unblinding, as participants reported whether they had noticed the magnetic strength of their bracelets (table 3). The overall pattern of results was replicated in the subgroup of 97 participants $(41(63 \%)$ in standard group $v 56(88 \%)$

Table 3 Participants' beliefs about magnet type and reasons given for beliefs. Values are numbers (percentages)

\begin{tabular}{lccc} 
& $\begin{array}{c}\text { Standard } \\
(\mathbf{n}=65)\end{array}$ & $\begin{array}{c}\text { Weak } \\
(\mathbf{n}=64)\end{array}$ & $\begin{array}{c}\text { Dummy } \\
(\mathbf{n}=64)\end{array}$ \\
\hline Belief about magnet: & & & \\
\hline Real & $35(54)$ & $24(38)$ & $10(16)$ \\
\hline Dummy & $5(8)$ & $12(19)$ & $30(47)$ \\
\hline Don't know & $22(34)$ & $23(36)$ & $22(34)$ \\
\hline Lost to follow up & $3(5)$ & $5(8)$ & $2(3)$ \\
\hline Reasons given*: & & & \\
\hline Condition improved & $14(22)$ & $10(16)$ & $8(13)$ \\
\hline Noticed magnetic force & $21(32)$ & $13(20)$ & $4(6)$ \\
\hline No change & 0 & $5(8)$ & $20(31)$ \\
\hline Condition worsened & $1(2)$ & $2(3)$ & $2(3)$ \\
\hline No reason/guessing & $4(6)$ & $5(8)$ & $4(6)$ \\
\hline Missing & 0 & $1(2)$ & $2(3)$ \\
\hline
\end{tabular}

*Participants who responded don't know were not asked for reasons, but percentages are of total in group. 
Table 4 Individual responses between baseline and 12 weeks

\begin{tabular}{|c|c|c|c|c|c|c|}
\hline & \multicolumn{2}{|c|}{ Standard magnet group ( $n=65)$} & \multicolumn{2}{|c|}{ Weak magnet group ( $n=64$ ) } & \multicolumn{2}{|c|}{ Dummy magnet group ( $n=64$ ) } \\
\hline & $\begin{array}{c}\text { No with high } \\
\text { pain at baseline }\end{array}$ & $\begin{array}{l}\text { No (\%) meeting } \\
\text { response criterion }{ }^{\dagger}\end{array}$ & $\begin{array}{l}\text { No with high } \\
\text { pain at baseline }\end{array}$ & $\begin{array}{l}\text { No (\%) meeting } \\
\text { response criterion }\end{array}$ & $\begin{array}{c}\text { No with high } \\
\text { pain at baseline }\end{array}$ & $\begin{array}{l}\text { No }(\%) \text { meeting } \\
\text { response criterion }\end{array}$ \\
\hline \multicolumn{7}{|l|}{ WOMAC A pain*: } \\
\hline Walking on flat surface & 51 & $22(34)$ & 55 & $16(25)$ & 54 & $18(28)$ \\
\hline Going up or down stairs & 63 & $19(29)$ & 63 & $12(19)$ & 62 & $11(17)$ \\
\hline At night in bed & 46 & $24(37)$ & 51 & $18(28)$ & 47 & $17(27)$ \\
\hline Sitting or lying & 44 & $24(37)$ & 43 & $22(34)$ & 42 & $16(25)$ \\
\hline Standing upright & 52 & $17(26)$ & 50 & $16(25)$ & 50 & $9(14)$ \\
\hline \multicolumn{7}{|l|}{ Visual analogue scale for paint: } \\
\hline High level of improvement & & $13(20)$ & & $9(14)$ & & $6(9)$ \\
\hline Moderate improvement & & $24(37)$ & & $27(42)$ & & $14(22)$ \\
\hline
\end{tabular}

${ }^{*}$ Criterion for meaningful change was shifting from categories of moderate, severe, or extreme pain to no or mild pain at week 12 compared with baseline. ${ }^{17}$ †OMERACT criteria for high and moderate levels of improvement in osteoarthritis trials (minimum $50 \%$ and $20 \%$ change from baseline respectively). ${ }^{20}$

in dummy group) who did not report noticing or testing the magnetic strength of their bracelets at week 12 . Results from analysis of covariance estimated the mean difference in WOMAC A between the standard and dummy groups as $1.3(95 \%$ confidence interval 0.003 to 2.62).

To examine the impact of the contamination of the weak magnets on the trial, we analysed data from only the bracelets that met the defined specification $(30$ weak magnets, 64 dummy magnets, and 64 standard magnets). Analysis of variance showed a significant difference for change in WOMAC A score $(\mathrm{F}=3.73$, $\mathrm{df}=2,155 ; \mathrm{P}=0.026$ ). The post-hoc Dunnett's test showed a significant difference between real and dummy magnets (mean difference 1.39, 95\% confidence interval 0.11 to 2.68), and a non-significant difference between real and weak magnets, although there was a strong numerical trend (mean difference $1.52,-0.09$ to $3.13, \mathrm{P}=0.067$ ).

Table 4 gives data on individual responses to treatments categorised according to predefined criteria for improvement. ${ }^{172}$ Participants' estimate of the monetary worth of the bracelet did not differ significantly. Adverse reactions were rare, with two participants in each group reporting dizziness, increased pain, or stiffness.

\section{Discussion}

We found evidence of a beneficial effect of magnetic wrist bracelets on the pain of osteoarthritis of the hip and knee. Self reported unblinding to treatment group did not substantially affect the results. Although there were problems with the weak magnets, a perspecification analysis suggested (but could not confirm) a specific effect of magnetic bracelets over and above placebo. Other reasons for suspecting a specific effect are that the data on belief show a low level of unblinding in the dummy group and the data on individual responses (table 4) show that more people achieve high levels of improvement in the standard magnet group. The results for two of the secondary outcome measures (WOMAC $\mathrm{C}$ and visual analogue pain scores) were consistent with this pattern. No change was seen in WOMAC B score, but this measure has been found to lack sensitivity. ${ }^{18}$

The findings are consistent with previous studies on magnetic therapies and pain. Studies that have failed to show an effect on pain ${ }^{10}{ }^{12}$ generally used weaker magnets (19 to 50 mTesla). Studies that have shown an effect used stronger magnets (47 to 180 mTesla), which were comparable with our standard strength magnets. ${ }^{24-8}$ Together these findings suggest that field strength is important.

\section{Is the effect real?}

Our study has not entirely resolved the extent to which the effect of magnetic bracelets is specific or due to placebo. Blinding did not affect the pattern of results, but the validity of the self reporting of blinding status could be questioned. Although the analysis of per-specification bracelets also suggests a specific effect, the result is only a trend and needs confirmation. Therefore, we cannot be certain whether our data show a specific effect of magnets, a placebo effect, or both.

Whatever the mechanism, the benefit from magnetic bracelets seems clinically useful. The mean reduction in WOMAC A scores in the intervention group of 2.9 (27\% change from baseline score) and the difference above placebo (1.3 points) is similar to that found in trials of frontline osteoarthritis treatments, including non-steroidal topical creams, ${ }^{21}$ oral nonsteroidal drugs (including cyclo-oxygenase 2 inhibitors) ${ }^{17}$ and exercise therapy. ${ }^{22}$ In a pivotal trial of cyclooxygenase 2 inhibitors ${ }^{17}$ in osteoarthritic patients with similar baseline pain (mean WOMAC A score 10.7) and the same follow up period (12 weeks), the treatment effects (change above placebo) were $0.8,1.5$, and 1.9 points for the three doses studied. The difference we found in physical function scores (WOMAC C) also compares well with the above trials. Furthermore, the effects seem additive to those of the participants' usual treatment. The (one off) cost of bracelets (around £30-£50 (\$58-\$96, €43-€92)), compares well with that of analgesics (paracetamol £20 a year, newer non-steroidal anti-inflammatories £250 a year). ${ }^{23}$ Larger investigations should now test the safety of magnets relative to the well known risks of analgesics. ${ }^{17} 2324$

The low refusal rate favours generalisability of our findings. However, the sample selected was predominantly white with a minimum WOMAC A score of 8 . Our results may thus not translate to other ethnic populations or people with milder osteoarthritis. Further work is needed to replicate our findings and determine whether the effect extends beyond 12 


\section{What is already known on this topic}

Static magnets are widely used for the relief of pain

Evidence about their efficacy in osteoarthritis is contradictory

Placebo effects are particularly difficult to control because of the easy detection of magnetism

\section{What this study adds}

Bracelets with static magnets decrease the pain from osteoarthritis of the hip and knee, over and above the effects of placebo

These benefits are supplementary to those from usual treatments

weeks. The contamination of group B with stronger magnets prevented a more objective estimation of anyplacebo effect. However, our design seems in principle a feasible way to allow for placebo effects in future studies.

We thank the Mid Devon general practices involved in the study and the research nurses. Mid Devon Primary Care Research Group provided support and advice throughout the study.

Contributors: TH had the original idea, led the writing, participated in planning, and was responsible for the overall management of study. CG and AW participated in planning and design, management of study (via steering group), and writing the manuscript. LB implemented the randomisation procedure and contributed to planning and design and writing the manuscript. $\mathrm{AH}$ took part in planning and design, analysis and interpretation, and writing the manuscript. EE participated in planning and design, management of study (via steering group), and writing. Mike Dixon took part in planning, design, and recruitment, and commented on the manuscript. Judith Mathie and Chris Rushton participated in management of study (via steering group), data collection, and coordination of trial nurses. Mark Taylor suggested the use of weak bracelets. All authors approved the final manuscript. EE is guarantor.

Funding: Arthritis Research Campaign.

Competing interests: None declared.

Ethical approval: North and East Devon local research ethics committee and West Somerset local ethics research committee.

1 Ecoflow. Single module magnetic bracelets. www.ecoflow.plc.uk/uk/ PeopleandAnimals/people_prod sin mod.htm (accessed 24 Feb 2004).

2 Weintraub M. Magnetic Bio-stimulation in painful diabetic peripheral neuropathy: a novel intervention-a randomized, double-placebo crossover study. Am J Pain Manage 1999;9:8-17.

3 Arthritis Research Campaign. Arthritis: the big picture. Chesterfield: ARC, 2002

4 Hinman R, Ford J, Heyl H. Effects of static magnets on chronic knee pain and physical function: A double-blind study. Altern Ther 2002;8(4):50-4.

5 Alfano AP, Taylor AG, Foresman PA, Dunkl PR, McConnell GG, Conaway $\mathrm{MR}$, et al. Static magnetic fields for treatment of fibromyalgia: a randomized controlled trial. J Altern Complement Med 2001;7:53-4.

6 Man D, Man B, Plosker H. The influence of permanent magnetic field therapy on wound healing in suction lipectomy patients: a double-blind study. Plast Reconstr Surg 1999;104:2267-8.

7 Brown CS, Ling FW, Wan JY, Pilla AA. Efficacy of static magnetic field therapy in chronic pelvic pain: a double-blind pilot study. Am J Obstet Gynecol 2002;187:1581-7.

8 Wolsko PM, Eisenberg DM, Simon LS, Davis RB, Wallaczek J, Mayo-Smith $\mathrm{M}$, et al. Double-blind placebo-controlled trial of static magnets for the treatment of osteoarthritis of the knee: results of a pilot study. Altern Ther 2004;10:36-43.

9 Weintraub MI, Wolfe GI, Barohn RA, Cole SP, Parry GJ, Hayat G, et al. Static magnetic field therapy for symptomatic diabetic neuropathy: a randomised, double blind, placebo-controlled trial. Arch Phys Med Rehabil 2003;5:736-46.

10 Collacott EA, Zimmerman JT, White PT, Rindone JP. Bipolar permanent magnets for the treatment of chronic low back pain. JAMA 2000; 283:1322-5.
11 Caselli MA, Clark N, Lazarus S, Velez Z, Venegas L. Evaluation of magnetic foil and PPT insoles in the treatment of heel pain. J Am Podiatr Med Assoc 1997;87:11-6.

12 Winemiller MH, Billow RG, Laskoswki ER, Harmsen WS. Effect of magnetic $v$ sham-magnetic insoles on plantar heel pain: a randomized controlled trial. JAMA 2003;290:45-56.

13 Bellamy N, Buchanan WW, Goldsmith CH, Campbell J, Stitt LW. Validation study of WOMAC: a health status instrument for measuring clinically important patient relevant outcomes to antirheumatic drug therapy in patients with osteoarthritis of the hip or knee. J Rheumatol 1988;15:1833-40.

14 Roos EM, Klassbo M, Lohmander LS. WOMAC osteoarthritis index Reliability, validity, and responsiveness in patients with arthroscopically assessed osteoarthritis. Scand J Rheumatol 1999:28:210-5.

15 Vallbona C, Richards T. Evolution of magnetic therapy from alternative to traditional medicine. Phys Med Rehabil Clin N Am 1999;10:729-54.

16 Price DD, McGrath PA, Rafii A, Buckingham B. The validation of the visual analogue scales as ratio scale measures for chronic and experimental pain. Pain 1983;17:45-56.

17 Zhao SZ, McMillen JI, Markenson JA, Dedhiva SD, Zhao WW, Osterhaus JT, et al. Evaluation of the functional status aspects of health-related quality of life of patients with osteoarthritis treated with celecoxib. Pharmacother 1999;19:1269-78.

18 Angst F, Aeschlimann A, Steiner W, Stucki G. Responsiveness of the WOMAC osteoarthritis index as compared with the SF-36 in patients with osteoarthritis of the legs undergoing a comprehensive rehabilitation intervention. Ann Rheum Dis 2001:60:834-40.

19 Schulz KF, Grimes DA. Blinding in randomised trials: hiding who got what. Lancet 2002;359:696-700.

20 Pham T, Van Der Heijde D, Lassere M, Altman RD, Anderson JJ, Bellamy $\mathrm{N}$, et al. Outcome variables for osteoarthritis clinical trials: the OMERACT-OARSI set of responder criteria. J Rheumatol 2003;30: 1648-54.

21 Bookman AAM, Williams KSA, Shainhouse JZ. Effect of a topical diclofenac solution for relieving symptoms of primary osteoarthritis of the knee: a randomized controlled trial. CMAJ 2004;171:333-8.

22 O'Reilly SC, Muir KR, Doherty M. Effectiveness of home exercise on pain and disability from osteoarthritis of the knee: a randomised controlled trial. Ann Rheum Dis 1999;58:15-9.

23 British Medical Association, Roval Pharmaceutical Society of Great Britain. British National Formulary. London: BMA, RPS, 2004. (No 47.)

24 Langman MJ. Adverse effects of conventional non-steroidal antiinflammatory drugs on the upper gastrointestinal tract. Fundam Clin Pharmacol 2003;17:393-403.

\section{Colourful new use for a word}

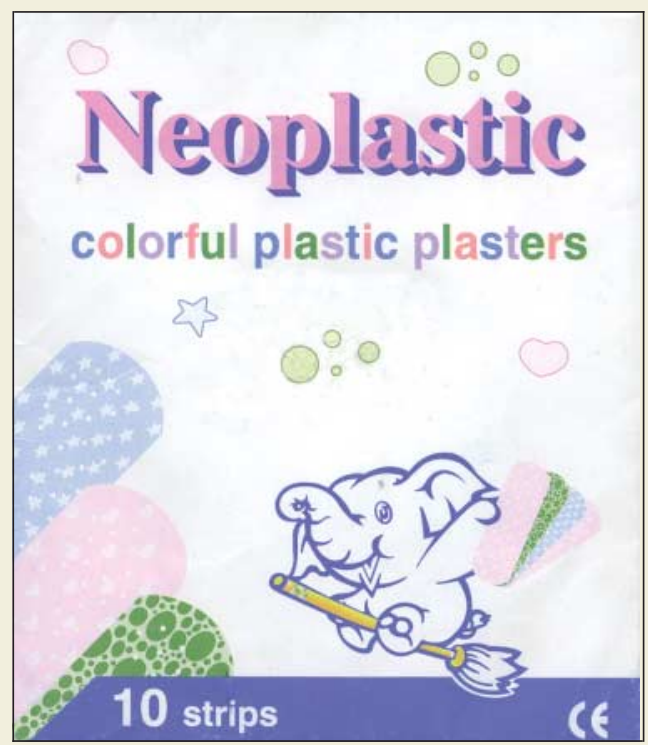

I bought these plasters in Malaysia; they are manufactured in Thailand.

Ian Bickle medical senior house officer,

Royal Victoria Hospital,Belfast (clonvara@yahoo.co.uk) 